\title{
Direct observation of multivalent states and $4 f \rightarrow 3 d$ charge transfer in Ce-doped yttrium iron garnet thin films
}

\author{
H. B. Vasili, ${ }^{1}$ B. Casals, ${ }^{2}$ R. Cichelero, ${ }^{2}$ F. Macià, ${ }^{2}$ J. Geshev,,${ }^{2,3}$ P. Gargiani, ${ }^{1}$ M. Valvidares, ${ }^{1}$ \\ J. Herrero-Martin, ${ }^{1}$ E. Pellegrin, ${ }^{1}$ J. Fontcuberta, ${ }^{2}$ and G. Herranz ${ }^{2}$ \\ ${ }^{1}$ CELLS-Divisió Experiments, ALBA Synchrotron Light Source, E-08290 Cerdanyola del Vallès, Barcelona, Catalonia, Spain \\ ${ }^{2}$ Institut de Ciència de Materials de Barcelona (ICMAB-CSIC), Campus de la UAB, E-08193 Bellaterra, Catalonia, Spain \\ ${ }^{3}$ Instituto de Física, Universidade Federal do Rio Grande do Sul, 91501-970 Porto Alegre, RS, Brazil
}

(Received 22 March 2017; revised manuscript received 15 June 2017; published 27 July 2017)

\begin{abstract}
Due to their large magneto-optic responses, rare-earth-doped yttrium iron garnets, $\mathrm{Y}_{3} \mathrm{Fe}_{5} \mathrm{O}_{12}$ (YIG), are highly regarded for their potential in photonics and magnonics. Here, we consider the case of Ce-doped YIG (Ce-YIG) thin films, in which substitutional $\mathrm{Ce}^{3+}$ ions are magnetic because of their $4 f^{1}$ ground state. In order to elucidate the impact of Ce substitution on the magnetization of YIG, we have carried out soft x-ray spectroscopy measurements on Ce-YIG films. In particular, we have used the element specificity of x-ray magnetic circular dichroism to extract the individual magnetization curves linked to $\mathrm{Ce}$ and $\mathrm{Fe}$ ions. Our results show that $\mathrm{Ce}$ doping triggers a selective charge transfer from Ce to the Fe tetrahedral sites in the YIG structure. This, in turn, causes a disruption of the electronic and magnetic properties of the parent compound, reducing the exchange coupling between the $\mathrm{Ce}$ and $\mathrm{Fe}$ magnetic moments and causing atypical magnetic behavior. Our work is relevant for understanding magnetism in rare-earth-doped YIG and, eventually, may enable a quantitative evaluation of the magneto-optical properties of rare-earth incorporation into YIG.
\end{abstract}

DOI: 10.1103/PhysRevB.96.014433

\section{INTRODUCTION}

In recent years, the ferrimagnet insulating yttrium iron garnet, $\mathrm{Y}_{3} \mathrm{Fe}_{5} \mathrm{O}_{12}$ (YIG), has received renewed attention owing to its remarkably small spin-wave damping, which makes it an excellent candidate for spintronics applications, exploiting phenomena such as the spin Seebeck effect [1] and spin pumping [2,3]. YIG is also well known to display a large magneto-optical response and low optical absorption and thereby is the material of choice for isolators and circulators in optical communications [4-6]. As a route to enhance the magneto-optical response, YIG is doped with rare earth elements, in particular, with cerium (Ce-YIG). Indeed, Ce doping on yttrium sites yields a significantly enhanced near-infrared Faraday rotation and magneto-optical figure of merit [7-11] without losing the magnetic insulator character. With these interesting properties, Ce-YIG is promising for nonreciprocal photonic device applications, such as the above-mentioned optical isolators and circulators [12,13]. Yet, despite the remarkable potential applications in optics, the fundamental physical mechanisms behind the enhanced magneto-optical response of Ce-YIG films are still being debated. Mostly, two types of electronic transitions are proposed, viz., either (i) intra-atomic orbital dipole transitions between the $4 f$ and $5 d$ states of $\mathrm{Ce}$ or (ii) interatomic dipole transitions between the $4 f$ states of $\mathrm{Ce}$ and $3 d$ states of $\mathrm{Fe}$ [7,14-16]. It is unclear, however, whether both transitions are involved or if one of them prevails over the other. Recently, we found that the individual contributions to the magnetism by $\mathrm{Ce}$ and $\mathrm{Fe}$ ions could be resolved by magneto-optical spectroscopy for particular spectral regions in the visible range [17]. We suggested that this result may offer a future possibility to clarify the role of the aforementioned electronic transitions in the optical response of Ce-YIG since the dynamic magnetic responses of $\mathrm{Ce}$ and $\mathrm{Fe}$ might yield distinctive optical signatures in ultrafast optical experiments that could shed light on the relevance of intrasite $\mathrm{Ce}^{3+}-\mathrm{Ce}^{3+}$ and intersite $\mathrm{Ce}^{3+}-\mathrm{Fe}^{3+}$ transitions [17].

Therefore, understanding the electronic structure of the $\mathrm{Ce} / \mathrm{Fe}$ cations and their specific contributions to the magnetism of doped YIG is a prerequisite for explaining the dipole transitions and related underlying physics behind the magneto-optical activity in the Ce-YIG systems. In view of this necessity, we have exploited the element-specific $\mathrm{x}$-ray absorption spectroscopy (XAS) and X-ray magnetic circular dichroism (XMCD) techniques on the Ce-YIG thin films to have direct access to the sublattice magnetizations of $\mathrm{Ce} / \mathrm{Fe}$ and to gather information on their valence states. In addition to element sensitivity, XMCD provides site-specific information on the magnetic properties. In this regard, it is useful to recall that yttrium iron garnet has a $\left\{\mathrm{Y}_{3}\right\}^{c}\left[\mathrm{Fe}_{2}\right]^{a}\left[\mathrm{Fe}_{3}\right]^{d} \mathrm{O}_{12}$ composition with a complex cubic structure (space group $\left.\mathrm{O}_{h}^{10}-I a \overline{3} d\right)$. As shown in Fig. 1(a), the nonmagnetic $\mathrm{Y}^{3+}$ ions occupy dodecahedral $(c)$ sites, while the $\mathrm{Fe}^{3+}$ ions occupy both tetrahedral $(d)$ and octahedral $(a)$ sites in the oxygen polyhedron structure. Figure 1(b) shows that in undoped YIG the Fe cations at $a$ and $d$ sites are coupled to each other antiferromagnetically by the superexchange interaction acting via the intervening $\mathrm{O}^{2-}$ anions. Finally, it is worth mentioning that magnetic anisotropy favors the orientation of the magnetization along the $\langle 111\rangle$ directions [18]. On the face of it, the study of orientation effects of applied magnetic fields is, at first glance, justified. As seen below, we achieved this purpose by growing films oriented along the $\langle 001\rangle$ and $\langle 111\rangle$ directions.

In this work, we anticipate that XAS and XMCD experiments provide important clues to understand the impact of Ce doping on both the electronic and magnetic properties of the $\mathrm{Ce}$-YIG system. For instance, our results reveal a charge transfer $\mathrm{Ce}^{3+}\left(4 f^{1}\right)+\mathrm{Fe}^{3+}\left(3 d^{5}\right) \rightarrow \mathrm{Ce}^{4+}\left(4 f^{0}\right)+\mathrm{Fe}^{2+}\left(3 d^{6}\right)$ taking place specifically at the tetrahedral $d$ sites of $\mathrm{Fe}$, 

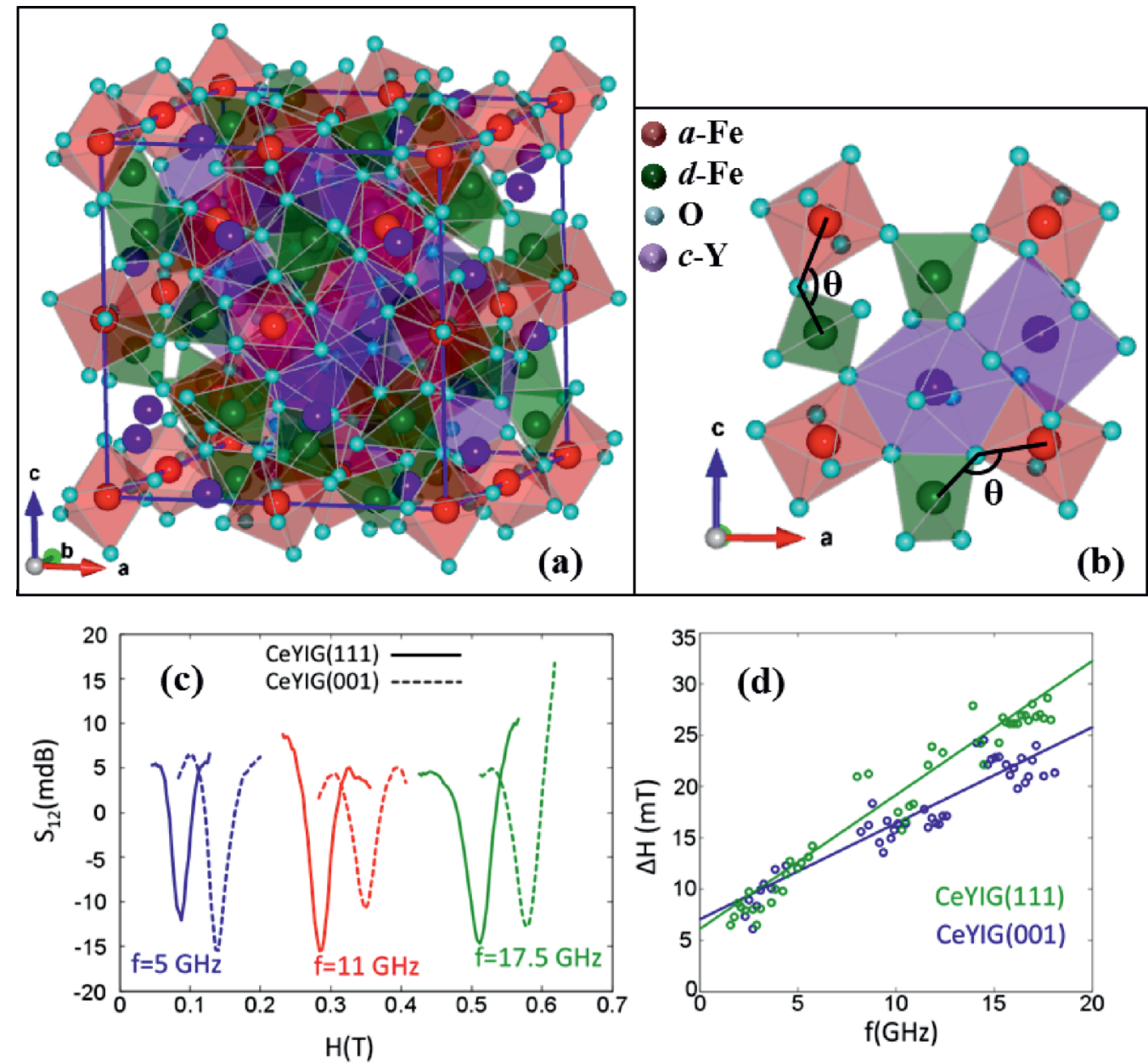

FIG. 1. (a) Schematic diagram of the YIG crystal structure. (b) Fe cations at $a$ and $d$ sites are connected to each other by a common corner of the oxygen anions. (c) FMR spectra of the (001)- and (111)- oriented Ce-YIG films for the frequencies $f=5,11$, and 17.5 GHz. (d) The FMR peak linewidths as a function of the resonance frequencies. Straight lines are the best fits based on Eq. (2) given in the text.

which causes a partial reduction of $\mathrm{Fe}^{3+}$ into $\mathrm{Fe}^{2+}\left(3 d^{6}\right)$. We demonstrate that the ensuing electronic changes alter the exchange coupling constant $J_{d / a}$ between $\mathrm{Fe}$ ions at $d$ and $a$ sites. We complete our study with analytic simulations, which consistently explain the $\mathrm{Ce}$ and $\mathrm{Fe}$ sublattice magnetizations in terms of the reduction of $J_{d / a}$, basically driven by the Ce doping. Briefly, our work offers an understanding of the multivalent cationic states and their site-specific contributions to the global magnetic response of doped YIG, which could pave the way towards better comprehension of the physical mechanisms that rule the optical responses in Ce-doped YIG systems.

\section{EXPERIMENTS}

Thin films of Ce-YIG $\left(\mathrm{CeY}_{2} \mathrm{Fe}_{5} \mathrm{O}_{12}\right)$ were grown on (001)and (111)-oriented gadolinium gallium garnet (GGG) substrates, and an undoped YIG film was grown on a GGG(111) substrate. The GGG substrates were chosen because of their best (i.e., lowest) lattice mismatch $(0.057 \%)$ possible for growing YIG/Ce-YIG thin films $[19,20]$. Details of the thinfilm growth process and the magneto-optic characterization of the above Ce-doped YIG films [54-nm Ce-YIG(001) and 56-nm Ce-YIG(111)] and the 200-nm-thick YIG (111) film were already discussed in our recent work [17]. To study further the magnetic dynamic properties, we performed ferromagnetic resonance (FMR) experiments on the above Ce-YIG films. For that purpose, we measured the transmission signal at different frequencies in a high-frequency broadband coplanar waveguide up to $20 \mathrm{GHz}$ using the flip-chip method.

To determine the site-specific magnetic contributions, XAS and XMCD measurements were carried out in total electron yield mode at room temperature at the BOREAS beamline of the ALBA Synchrotron light source [21]. For Ce, the spectra were taken at the $M_{4,5}$ absorption edges, while for Fe the spectra were recorded at the $L_{2,3}$ absorption edges. XMCD spectra were obtained by taking the difference between the XAS of right $\left(\sigma^{+}\right)$and left $\left(\sigma^{-}\right)$circularly polarized light. In the XMCD measurements, the samples were magnetized by applying a magnetic field $H$ of $2 \mathrm{~T}$ along the outof-plane direction of the films (along the beam direction). For the XMCD data analysis, we have calculated model XMCD spectra using the CTM4XAS 5.5 program, which is based on crystal-field multiplet calculations, including the 
Slater integrals, crystal-field parameters (crystal symmetry, $10 D q$, and magnetic energy), and spin-orbit coupling [22]. Site-specific magnetization $M$ vs $H$ curves were measured by selecting the photon energies at the XMCD peaks of Fe ions at $a$ sites, Fe ions at $d$ sites, and Ce ions at the $c$ sites at the corresponding photon energies. To obtain the above XMCD hysteresis cycles, $H$ was varied between $+2 \mathrm{~T}$ and $-2 \mathrm{~T}$, and the polarization of light was successively switched from leftto right-handed circularly polarized light.

\section{RESULTS AND DISCUSSION}

Before discussing the X-ray spectroscopy results and the subsequent magnetization modeling, it is relevant to have a brief discussion on our structural and dynamic magnetic characterizations of the Ce-YIG thin films. With this in mind, we have verified the crystalline structure of the Ce-doped YIG films and the undoped YIG film by x-ray diffraction (XRD) measurements where the samples were shown to have a high crystallinity with good epitaxy. We discuss the XRD results in the Supplemental Material [23]. In particular, our analysis did not reveal any secondary $\mathrm{CeO}_{2}$ phases in Ce-doped YIG films, which has been reported to arise for high concentrations of Ce [24,25]. Additionally, we were able to observe Laue oscillations that can be appreciated in $\theta-2 \theta$ scans, indicating good epitaxy of the Ce-YIG samples.

To obtain information on the spin dynamics of Ce-YIG films we performed FMR experiments in which we measured the transmitted microwave power in a coplanar waveguide placed on top of the films. A nominal microwave power of $0 \mathrm{dBm}$ was used for the experiments, and the frequencies were in the range of 1 to $18 \mathrm{GHz}$. The microwave-absorbed signal was recorded while an applied magnetic field from -0.6 to $0.6 \mathrm{~T}$ was swept at each frequency. In Fig. 1(c) we plot representative curves of the amplitude of transmission coefficients $S_{12}$ as a function of the magnetic field for different frequencies $(5,11$, and $17.5 \mathrm{GHz})$ on both (001)- and (111)oriented Ce-YIG thin films. These curves were fitted using the Lorentz equation

$$
S_{12}(H) \propto\left[\frac{(\Delta H)^{2}}{\left(H_{\mathrm{FMR}}-H\right)^{2}+(\Delta H)^{2}}\right],
$$

where $H_{\mathrm{FMR}}$ is the magnetic field under the resonance condition and $\Delta H$ is the half linewidth. Figure 1(d) shows the parameter $\Delta H$ extracted from the fittings as a function of the excitation frequencies. We thus determine the Gilbert damping by fitting the data (straight lines) using the following equation [26]:

$$
\Delta H=\Delta H_{0}+\left(\frac{2 \pi}{\gamma}\right) \alpha f,
$$

where $\gamma$ is the gyromagnetic ratio $[\gamma /(2 \pi)=28 \mathrm{GHz} / \mathrm{T}], \alpha$ is the Gilbert damping, and $\Delta H_{0}$ is the inhomogeneous damping of the films. We have obtained a good estimation of the Gilbert damping values of $\alpha=0.026$ and $\alpha=0.036$ for the (001) and (111) orientations, respectively. In fact, the above values are very close to those reported previously for Ce-YIG thin films [27]. The slightly larger damping of the (111)-oriented film might be related to some magnetic anisotropy induced during growth. As discussed by Kehlberger et al. [27], this observation is supported by the different strain states of the (111)- and (001)-oriented films, plausibly leading to a magnetoelastic contribution to the magnetic anisotropy.

We also notice that the damping values of Ce-YIG films are considerably larger than those usually reported for undoped YIG films, which are on the order of $10^{-4}$ [27]. As discussed previously by other authors [27], this reduction of about two orders of magnitude can be explained from various contributions, such as lattice dilation and different environments of the $\mathrm{Fe}$ cations due to the Ce doping. These observations are also in agreement with the values that we obtain for the inhomogeneous damping since our analysis yields values around $\Delta H_{0} \approx 5 \mathrm{mT}$, which are considerably larger than in undoped YIG films $(<0.5 \mathrm{mT})$ [27]. We stress, however, that despite the higher damping values in Ce-YIG films, the damping parameters are comparable to those of most of the metallic ferromagnetic thin films [28,29].

To probe further into the individual $\mathrm{Ce} / \mathrm{Fe}$ sublattice environments, we looked into $\mathrm{X}$-ray spectroscopy characterizations of the YIG and Ce-YIG thin films. We discuss first the oxidation states of $\mathrm{Ce}$ and $\mathrm{Fe}$ as derived from the analysis of the XAS and XMCD spectra. To start with, Figs. 2(a) and 2(b) show the Ce $M_{4,5}$ XAS spectra of the (001)- and (111)-oriented Ce-YIG thin films, respectively, obtained under illumination of the sample with $\sigma^{+}$and $\sigma^{-}$circularly polarized light at normal incidence. After normalizing the raw XAS signal with the gold mesh signal, the spectra were further renormalized to unity at the postedge $(912 \mathrm{eV})$ of the $M_{4}$ peak. We first draw the attention to the fine structure in the Ce $M_{4,5}$ spectra, which arises from the multiplet structure of the final states of the $\mathrm{Ce}^{3+}\left(4 f^{1}\right)$ and $\mathrm{Ce}^{4+}\left(4 f^{0}\right)$ ground-state configurations. Different spectral signatures can be observed in the XAS spectra, i.e., contributions from $\mathrm{Ce}^{3+}\left(f^{1}\right), \mathrm{Ce}^{4+}\left(f^{0}\right)$, and $\mathrm{Ce}^{4+}\left(f^{1} L\right)$ states, as shown in Fig. 2(a). These signatures enable the identification of the $\mathrm{Ce}$ valence states, clearly visible in the experimental XAS data of pure $\mathrm{Ce}_{2} \mathrm{O}_{3}\left(\mathrm{Ce}^{3+}\right)$ and $\mathrm{CeO}_{2}\left(\mathrm{Ce}^{4+}\right)$ as extracted from [30] [see Fig. 2(c)]. As a consequence, we have used these data to quantify the relative amount of $\mathrm{Ce}^{3+}$ and $\mathrm{Ce}^{4+}$ in our Ce-YIG(001) and Ce-YIG(111) films. Total XAS spectra $\left(\sigma^{+}+\sigma^{-}\right)$of the Ce $M_{4,5}$ recorded from our samples were then fitted to the reference spectra shown in Fig. 2(c). Such a fitting of the total XAS is shown in Fig. 2(d) for the Ce-YIG(001) film. From the comparison of spectral weights, shown in Fig. 2(d), we could estimate that the fraction of $\mathrm{Ce}^{3+}$ is approximately $30 \%$, while the remaining Ce cations are in the $\mathrm{Ce}^{4+}$ state.

Since Ce ions are expected to replace Y in the YIG structure, this raises a question about the valence states of yttrium ions. To address this issue, we have measured the $\mathrm{Y} L_{2,3} \mathrm{XAS}$ and XMCD for both undoped YIG and Ce-YIG(001) samples. Our results confirm that yttrium is in only the $\mathrm{Y}^{3+}$ valence state and the XAS and XMCD spectral features are very similar to those reported by Rogalev et al. [31] for YIG thin films. More details of the XAS and XMCD data are given in the Supplemental Material [23].

On the other hand, to unveil the valence states of iron, we analyzed the prepeak structure of the $L_{3}$ edge (peaks A and B) and the double-peak structure (peaks $\mathrm{C}$ and D) of the $L_{2}$ edge in the total Fe $L_{2,3}$ XAS spectra of the Ce-YIG films as well as the undoped YIG film [see the inset of Fig. 3(a)]. These multiplet analyses could be used as a proxy to determine the valence state 

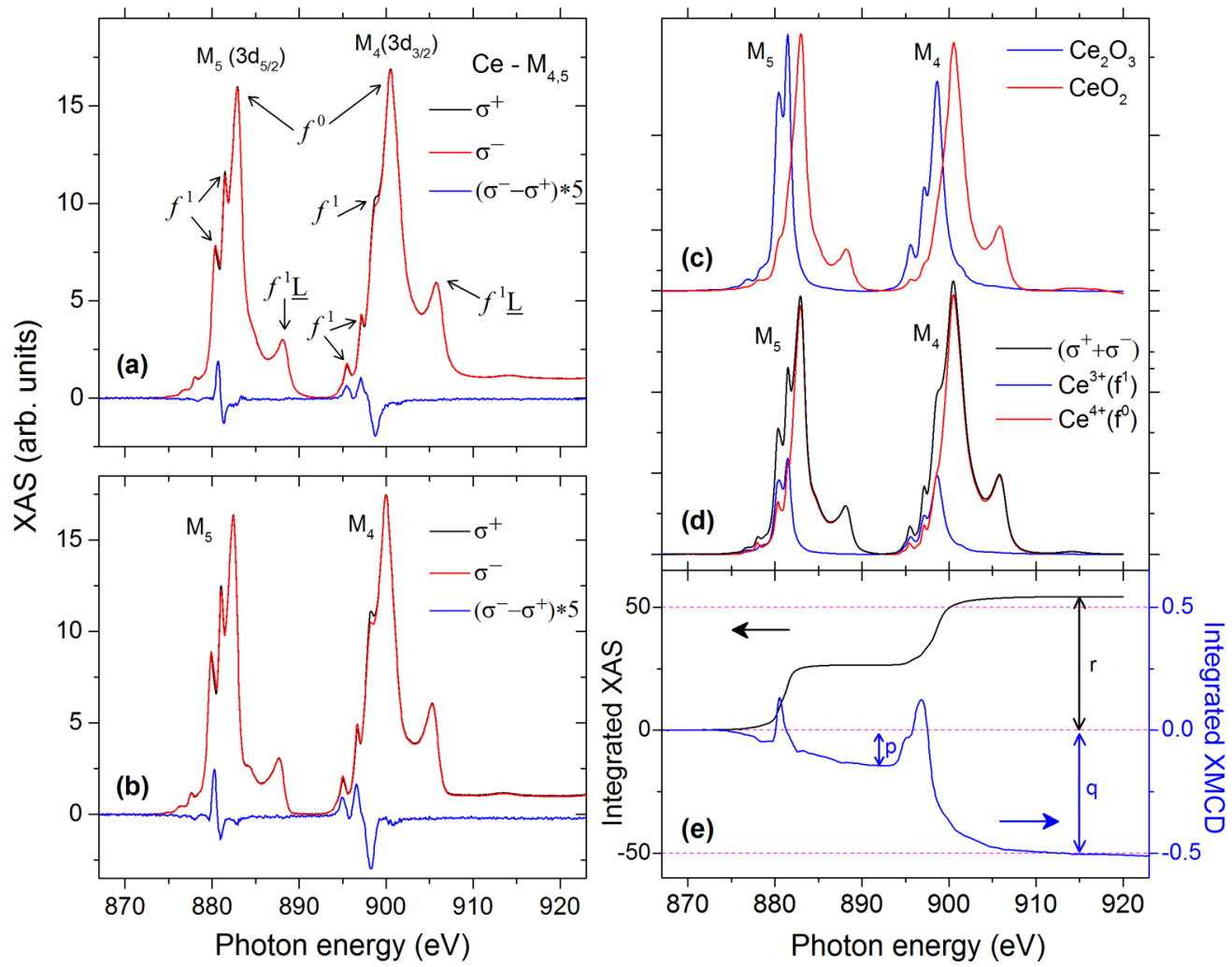

FIG. 2. Ce-M $\mathrm{M}_{4,5} \mathrm{XAS}$ and XMCD of (a) Ce-YIG(001) and (b) Ce-YIG(111) thin films at $300 \mathrm{~K}$. The spectral features of mixed Ce ${ }^{3+}\left(f^{1}\right)$ and $\mathrm{Ce}^{4+}\left(f^{0}, f^{1} \mathrm{~L}\right)$ ground state contributions are well understood from the experimental Ce-M $\mathrm{M}_{4,5}$ XAS data extracted from Ref. [30], given in (c). The XMCD $\left(\sigma^{-}-\sigma^{+}\right)$in panels (a) and (b) arises mainly from the $\mathrm{Ce}^{3+}$ contribution. (d) The Ce $\mathrm{Ce}^{3+}$ XAS was separated out by fitting the experimental data to the extracted data. Panel (e) shows integration of the $\mathrm{Ce}^{3+} \mathrm{XAS}$ and XMCD spectra. A detailed sum-rule analysis is given in the text.

of Fe. The $L_{2,3}$ edge spectral features observed in our undoped YIG film [Fig 3(a)] are those typical of $\mathrm{Fe}^{3+}$, similar to, e.g., $\gamma-\mathrm{Fe}_{2} \mathrm{O}_{3}$ structure [32], in which the A/B peak ratio is $\sim 0.46$. On the contrary, as seen in Figs. 3(b) and 3(c), the Ce-YIG films display significantly higher $\mathrm{A} / \mathrm{B}$ ratio $[\sim 0.62$ and $\sim 0.70$ for (001) and (111)-oriented films, respectively]. Therefore, the evolution of the $\mathrm{A} / \mathrm{B}$ ratio with $\mathrm{Ce}$ doping indicates the emergence of a $\mathrm{Fe}^{2+}$ oxidation state [32,33]. In particular, the growing preedge $\mathrm{Fe} L_{2}$ feature [indicated by arrows in the inset of Fig. 3(a)] is often regarded as the presence of $\mathrm{Fe}^{2+}$ in the system [32,33]. Therefore, the analysis of our XAS spectra shows that doping YIG with Ce induces the concurrent emergence of $\mathrm{Fe}^{2+}$ and $\mathrm{Ce}^{4+}$ valence states, which gives evidence for a charge transfer from $\mathrm{Ce}$ to $\mathrm{Fe}$. Indeed, $\mathrm{Ce}^{4+}$ states have been reported for other Ce oxide structures, and their origin has been attributed to the hybridization between Ce $4 f$ and neighboring $\mathrm{O} 2 p$ or transition-metal $3 d$ bands $[34,35]$. From first-principles calculations reported for the Ce-YIG system, it is known that the charge transfer takes place from $\mathrm{Ce}(4 f)$ to $\mathrm{Fe}(3 d)$ states at $d$-(tetrahedral) sites [14]. In the following, we show that this prediction is confirmed by the analysis of our XMCD spectra and further supported by multiplet XMCD calculations.

First, we note that the XMCD $\left(\sigma^{-}-\sigma^{+}\right)$spectrum of the undoped YIG film shows two positive peaks caused by $\mathrm{Fe}^{3+}$ ions at octahedral $a$ sites [labeled $a_{1}$ and $a_{2}$ in Figs. 3(a) and $3(\mathrm{e})]$ and one large negative peak for tetrahedral $d$ sites. We have simulated these XMCD spectra by ligand field multiplet (LFM) calculations [36] on $a-\mathrm{Fe}^{3+}$ and $d \mathrm{Fe}^{3+}$ sites and found a very good agreement with the above experimental spectra [see Fig. 3(d)]. This observation confirms that Fe ions in the undoped YIG film are in an $\mathrm{Fe}^{3+}$ state. However, the XMCD spectra of Ce-YIG films, normalized to both $a$-site peaks, show a prominent extra peak at an energy $(707.5 \mathrm{eV})$ below the $d$-site feature [see Fig. 3(e)]. This extra peak, which can also be reproduced by our multiplet calculations, is direct evidence of the aforementioned presence of $\mathrm{Fe}^{2+}$ ions, specifically at $d$ sites in the Ce-doped YIG films. Further indication of specific charge transfer to $d$ sites can be inferred from changes in the ratio between the $d$ - and $a$-site peak intensities of the Fe $L_{3}$ XMCD spectra. In particular, the intensities of $a_{1}$ and $a_{2}$ peaks remain unchanged in the normalized XMCD [see Fig. 3(e)], which rules out any charge transfer to the $a$ sites. On the contrary, our XMCD results confirm that the charge transfer occurs to the tetrahedral $d\left(\mathrm{Fe}^{2+}\right)$ sites since the calculated intensity ratio between the $d$ peak $(708.3 \mathrm{eV})$ and the $a_{2}$ peak $(708.9 \mathrm{eV})$ is smaller in Ce-YIG films than for the undoped YIG film.

To investigate the impact of Ce doping on the YIG magnetic properties, we have evaluated the magnetic moments of $\mathrm{Ce}$ and $\mathrm{Fe}$ ions in the Ce-YIG films as well as the Fe moments in the undoped YIG film. Concerning Ce, our XMCD spectra reveal that the magnetism arises mainly from the localized $4 f^{1}$ electrons $\left(\mathrm{Ce}^{3+}\right)$, while the $4 f^{0}\left(\mathrm{Ce}^{4+}\right)$ ground state shows a 

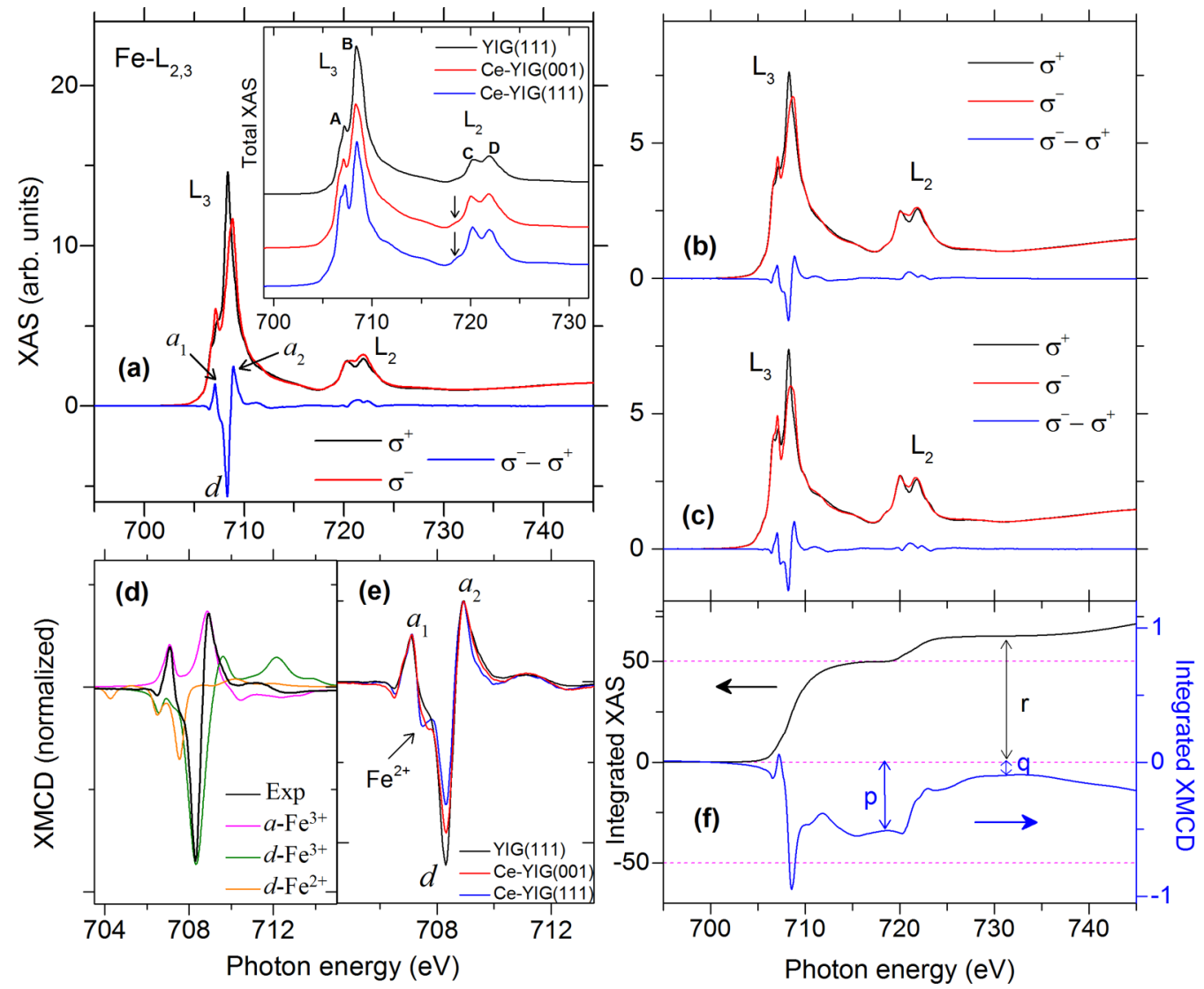

FIG. 3. Fe- $\mathrm{L}_{2,3} \mathrm{XAS}$ and XMCD of (a) YIG(111), (b) Ce-YIG(001), and (c) Ce-YIG(111) thin films at $300 \mathrm{~K}$. Total XAS $\left(\sigma^{-}+\sigma^{+}\right)$in the inset (a) reveals that the YIG film has only $\mathrm{Fe}^{3+}$ while the $\mathrm{Ce}$-YIG films exhibit an additional $\mathrm{Fe}^{2+}$ contribution. The $\mathrm{A} / \mathrm{B}$ peak ratio along with the growing $L_{2}$ pre-edge feature (shown by arrows) hints at the presence of $\mathrm{Fe}^{2+}$ in the films. Panel (d) shows the calculated XMCD spectra for $a-\mathrm{Fe}^{3+}, d-\mathrm{Fe}^{3+}$, and $d-\mathrm{Fe}^{2+}$ contributions. From this, it appears that the Fe- $\mathrm{L}_{3}$ XMCD of YIG film has two positive peaks for $a$ sites and a large negative peak for $d$ sites. Panel (e) shows a growing extra XMCD peak (shown by arrow) that confirms the Fe ${ }^{2+}$ in the Ce-YIG films. Panel (f) shows the integration of Fe-XAS and XMCD spectra of YIG film. Please refer to the text for the sum-rule analysis.

considerably smaller dichroic effect and the $4 f^{1} L$ ground state exhibits no contribution to the magnetism, as seen in Figs. 2(a) and 2(b). Consequently, to evaluate the magnetic moment of $\mathrm{Ce}$ we have separated out the $\mathrm{Ce}^{3+}$ contribution from the total XAS spectra by fitting the $\mathrm{Ce}^{3+}$ and $\mathrm{Ce}^{4+} \mathrm{XAS}$ data of Fig. 2(c). The spin $\left(m_{s}\right)$ and orbital $\left(m_{l}\right)$ components of atomic magnetic moments per YIG and Ce-YIG formula unit were obtained by applying the sum rules to the integrated intensities of total XAS and XMCD spectra [37-39]. The results of the analysis are summarized in Table I. For the sum rules, the electric dipole transitions of an ion are considered from a spin-orbit-split core level to the shell-resolved operators of the valence electrons. The sum rule equations for Fe $L_{2,3}$ $(2 p \rightarrow 3 d)$ and Ce $M_{4,5}(3 d \rightarrow 4 f)$ absorption edges are given by

$$
2\left\langle S_{z}\right\rangle+7\left\langle T_{z}\right\rangle=\left(\frac{6 p-4 q}{r}\right) N_{h}, \quad\left\langle L_{z}\right\rangle=\left(\frac{4 q}{3 r}\right) N_{h}
$$

for the $2 p \rightarrow 3 d$ dipole transitions and

$$
2\left\langle S_{z}\right\rangle+6\left\langle T_{z}\right\rangle=\left(\frac{5 p-3 q}{r}\right) N_{h}, \quad\left\langle L_{z}\right\rangle=\left(\frac{2 q}{r}\right) N_{h}
$$

for the $3 d \rightarrow 4 f$ transitions. In Eqs. (3) and (4), $r$ refers to the integrated value of the total XAS (after subtracting the step function) at the $L_{2}$ and $M_{4}$ postedges, as shown in Figs. 3(f) and 2(e), respectively. The parameters $p$ and $q$ refer to the integrated values of the XMCD at $L_{3}\left(M_{5}\right)$ and $L_{2}\left(M_{4}\right)$ postedges, respectively [see Figs. 3(f) and 2(e)]. $\left\langle L_{z}\right\rangle,\left\langle S_{z}\right\rangle$, and $\left\langle T_{z}\right\rangle$ are the expectation values with respect to the $z$ component of the orbital angular momentum, the spin angular momentum, and the magnetic dipole operator of the $3 d(4 f)$ shell for $L_{2,3}$ $\left(M_{4,5}\right)$ edges, respectively. $N_{h}$ is the number of holes, taken to be 4.7 for the $3 d^{5}$ shell [40] and 13 for the $4 f^{1}$ shell in the calculations. Using the above equations, one can estimate the orbital and spin magnetic moments as $m_{l}=-\left\langle L_{z}\right\rangle \mu_{\mathrm{B}}$ and $m_{s}=-2\left\langle S_{z}\right\rangle \mu_{\mathrm{B}}$, respectively. The derived moments of the YIG and Ce-YIG films are listed in Table I.

A few comments about the evaluation of the magnetic moments are relevant. In the first place, corrections to the spin sum rules were taken into account for the $\mathrm{Fe}^{3+}$ values given in Table I, as it is well known that strong deviations are caused by core-valence exchange interactions in the transition-metal ions [41]. Additionally, the values of $\left\langle S_{z}\right\rangle$ for $\mathrm{Fe}^{3+}$ were determined by assuming that $\left\langle T_{z}\right\rangle$ is negligible due to the cubic symmetry of the $d$ orbitals. However, the assumption of negligible $\left\langle T_{z}\right\rangle$ does not hold for rare-earth ions like Ce ions because of the localized and aspherical $4 f$ electron charge densities. For these ions, the magnitude of $\left\langle T_{z}\right\rangle$ is indeed comparable to that of $\left\langle S_{z}\right\rangle$, 
TABLE I. Effective spin $\left(2\left\langle S_{z}\right\rangle+n\left\langle T_{z}\right\rangle\right)$ and orbital $\left(\left\langle L_{z}\right\rangle\right)$ magnetic moments of the YIG and Ce-YIG films per YIG and Ce-YIG formula unit calculated using the XMCD sum rules on Ce-M and Fe-L absorption edges. Here, $n=6$ for Ce $M$ edges, and $n=7$ for Fe $L$ edges. The $m_{s}$ of $\mathrm{Ce}$ and tetrahedral $\mathrm{Fe}$ ions are coupled antiferromagnetically with Ce carrying a very small $m_{s}$ value compared to that of Fe. The parameter $m_{\text {tot }}$ is defined as the total effective spin and orbital magnetic moments. The Ce-YIG films exhibit a smaller $m_{\text {tot }}$ than the undoped YIG film.

\begin{tabular}{|c|c|c|c|c|c|c|}
\hline Sample & Absorption edge & $2\left\langle S_{z}\right\rangle+n\left\langle T_{z}\right\rangle /$ f.u. $\left(\mu_{\mathrm{B}}\right)$ & $2\left\langle S_{z}\right\rangle /$ f.u. $\left(\mu_{B}\right)$ & $\left\langle L_{z}\right\rangle /$ f.u. $\left(\mu_{B}\right)$ & $m_{\text {tot }} /$ f.u. $\left(\mu_{\mathrm{B}}\right)$ & $\left|\frac{\left\langle L_{z}\right\rangle}{2\left\langle S_{z}\right\rangle}\right|$ \\
\hline \multirow{2}{*}{ Ce-YIG(001) } & Ce $M$ & -0.191 & -0.033 & 0.243 & 0.052 & 7.379 \\
\hline & $\mathrm{Fe} L$ & 1.461 & 1.461 & 0.050 & 1.511 & 0.034 \\
\hline \multirow{4}{*}{ Ce-YIG(111) } & & & & & $m_{\mathrm{tot}}=1.563$ & \\
\hline & Ce $M$ & -0.214 & -0.037 & 0.280 & 0.066 & 7.589 \\
\hline & $\mathrm{Fe} L$ & 1.320 & 1.320 & 0.045 & 1.365 & 0.034 \\
\hline & & & & & $m_{\mathrm{tot}}=1.431$ & \\
\hline YIG(111) & $\mathrm{Fe} L$ & 2.558 & 2.558 & 0.017 & 2.575 & 0.006 \\
\hline
\end{tabular}

and the former cannot be determined directly. In order to have an estimation of the spin-only component of the Ce magnetic moment, we assumed that $\left\langle T_{z}\right\rangle$ is proportional with $\left\langle S_{z}\right\rangle$, as demonstrated in [42]. The ratio $\frac{\left\langle T_{z}\right\rangle}{\left\langle S_{z}\right\rangle}\left(=\frac{8}{5}\right)$ for $\mathrm{Ce}^{3+}$ was then calculated using the theoretical expectation values for $\left\langle T_{z}\right\rangle$ and $\left\langle S_{z}\right\rangle$ from the Hund's rule states given in [43]. Using the above ratio, we have extracted the $2\left\langle S_{z}\right\rangle$ values from $2\left\langle S_{z}\right\rangle+6\left\langle T_{z}\right\rangle$ of the Ce $M$ edges (see Table I). The consistency of these assumptions is shown by noting that for $\mathrm{Ce}^{3+}\left(4 f^{1} ; S=1 / 2\right.$, $L=3)$ we should expect $m_{l} / m_{s}=[L(L+1) / 2 S(S+1)]=$ 8. Our experimental values of $\left|m_{l} / m_{s}\right|$ are indeed close to 8 , confirming the above-mentioned assumptions. Furthermore, the moments in Table I reveal that $m_{s}$ of $\mathrm{Fe}$ and $\mathrm{Ce}$ are antiparallel to each other [see the negative (positive) signs for $m_{s}$ of $\mathrm{Ce}(\mathrm{Fe})$ in Table I]. We note also that the $\mathrm{Y}^{3+}$ ions exhibit almost negligible magnetic moments $\left(<0.01 \mu_{\mathrm{B}}\right)$, and therefore, they were not considered for evaluating $m_{\text {tot }}$ values (more details are given in the Supplemental Material [23]). Note that $m_{\text {tot }}$ of the YIG film $\left(2.57 \mu_{\mathrm{B}}\right)$ is reduced from $5 \mu_{\mathrm{B}}\left(\mathrm{Fe}^{3+}\right.$ free-ion moment) for the YIG structure. In the YIG structure, the ordered magnetic moment decreases mono- tonically with the increasing temperature and vanishes at the Curie temperature $T_{\mathrm{C}}$ [44]. The undoped YIG(111) film in our study has a bulklike magnetic moment $m_{\text {tot }}$ of $4.31 \mu_{\mathrm{B}}$ at $2 \mathrm{~K}$. However, as commonly observed for YIG epitaxial films or bulk $[45,46]$, the moment at room temperature is substantially decreased, to about $2.57 \mu_{\mathrm{B}}$ in our case (see Table I).

From Table I we see also that the Ce-doped YIG films exhibit significantly smaller $m_{\text {tot }}$ values than the undoped YIG film, indicating that Ce doping affects the magnetization of the Fe sublattice. In principle, this is expected from the fact that the Ce doping induces a charge transfer on the Fe sites, so that $\mathrm{Fe}^{2+}$ valence states emerge, thereby causing a reduction in the nominal $\mathrm{Fe}(d)$ spin magnetic moment from $S=5 / 2$ to $S=4 / 2$. Additionally, this also produces concomitant changes in the antiferromagnetic coupling between the $a(\mathrm{Fe})$ and $d(\mathrm{Fe})$ sites. To gain insight into this antiferromagnetic exchange coupling, we have studied the site-specific magnetic contributions of $d$ $\mathrm{Fe}, a \mathrm{Fe}$, and $c \mathrm{Ce}$ and their evolution with magnetic field. For that purpose, we exploited the site-dependent XMCD magnetization curves of the undoped YIG as well as the Ce-doped YIG films (see Fig. 4). For a better visualization, the

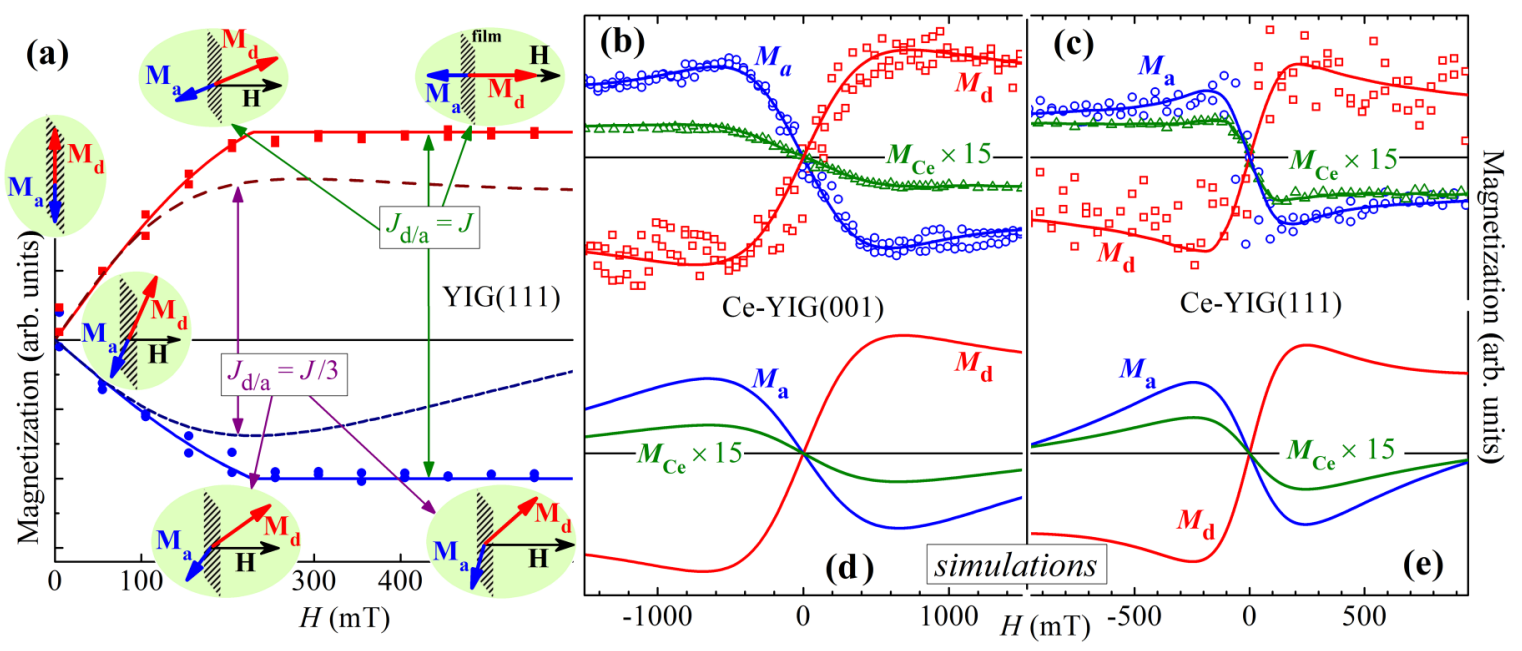

FIG. 4. (a) Out-of-plane XMCD (symbols) and model (solid lines) magnetization curves of $d$ and $a$ sites of YIG(111) film given in the upper and lower side of the panel, respectively. For the sake of clarity, only the positive field range is plotted. The evolution of magnetizations in the $d$ and $a$ sites $\left(\mathbf{M}_{d}\right.$ and $\mathbf{M}_{a}$, respectively) is given for the $\mathbf{M}_{d} / \mathbf{M}_{a}$ antiparallel coupling ( $\left.J_{d / a}\right)$ in two cases: (i) $J$ (top sketches) for YIG film and (ii) $J / 3$ (bottom sketches) for the Ce-YIG films. The solid lines in the XMCD hysteresis data of (b) Ce-YIG(001) and (c) Ce-YIG(111) films are given for a guided view. Panels (d) and (e) represent the simulated magnetization loops for (001)- and (111)-oriented Ce-doped YIG films, respectively. 
curves in Fig. 4(a) are shown only in the positive field range and were obtained by selecting the XMCD peak energies at $d$ sites $(708.2 \mathrm{eV})$ and $a$ sites $(708.9 \mathrm{eV})$. Drawing attention first to the undoped YIG film in Fig. 4(a), we see that the measured $\mathrm{Fe} L_{3}$ XMCD signal saturates at $H \approx 200 \mathrm{mT}$. However, the Fe $L_{3}$ XMCD magnetization curves of (001)- and (111)-oriented films of Ce-doped YIG show deviations from the conventional saturation-magnetization behavior at $H \approx$ $500 \mathrm{mT}$ [Fig. 4(b)] and $H \approx 200 \mathrm{mT}$ [Fig. 4(c)], respectively. More precisely, in contrast to the undoped YIG film, where the magnetization, once saturated, remains constant [see Fig. 4(a)], the hysteresis curves of Ce-YIG films exhibit a small magnetization overshoot at the fields indicated above. These atypical magnetization curves are reminiscent of those that we obtained previously in our magneto-optical spectroscopy experiments [17].

In order to find an explanation for this unconventional behavior, the XMCD loops were interpreted through numerical simulations performed using a phenomenological model. The model incorporates as inputs the normalized values of the XMCD loops, in which the Fe spins of $d$ sites $\mathbf{M}_{\boldsymbol{d}}$ are antiferromagnetically coupled (via superexchange) to those at $a$ sites $\mathbf{M}_{\boldsymbol{a}}$ as well as to the $\mathrm{Ce}^{3+}$ spins in the $c$ sites $\mathbf{M}_{\mathbf{C e}}$. We assume that the magnetic energy density $E$ for $\mathbf{H}$ applied along the out-of-plane direction to the film surface is given by

$$
\begin{aligned}
E= & \sum_{i}^{d, a} K_{i}^{\mathrm{eff}}\left(\frac{\mathbf{M}_{\boldsymbol{i}} \cdot \mathbf{n}}{M_{i}}\right)^{2}-\sum_{i}^{d, a, \mathrm{Ce}} \mathbf{H} \cdot \mathbf{M}_{i} \\
& +\sum_{i \neq j}^{d, a, \mathrm{Ce}} J_{i / j}\left(\frac{\mathbf{M}_{i} \cdot \mathbf{M}_{j}}{M_{i} M_{j}}\right) .
\end{aligned}
$$

The first term in Eq. (5) represents the in-plane magnetic anisotropies of $\mathbf{M}_{\boldsymbol{d}}$ and $\mathbf{M}_{\boldsymbol{a}}$ (where the effective anisotropy constants $K_{d}^{\text {eff }}$ and $K_{a}^{\text {eff }}$ result from the shape and any other anisotropy contributions and the unit vector $\mathbf{n}$ gives the out-ofplane direction). The second term adds the Zeeman energies of all magnetic contributions, whereas the last term takes into account all the effective coupling energies parameterized by the $J_{i / j}$ constants, representing the interaction between $i$ and $j$ sites. Since the $\mathrm{Ce}^{3+}$ ions have small magnetic moments (see Table I), we have assumed that the $\mathrm{Ce}^{3+}$ anisotropy is negligible. The values of the magnetizations of each sublattice at any value of $H$ are obtained by minimizing $E$ with respect to the equilibrium states of $\mathbf{M}_{\boldsymbol{d}}, \mathbf{M}_{\boldsymbol{a}}$, and $\mathbf{M}_{\mathbf{C e}}$. More details on the energy minimization procedure employed here can be found in Refs. [47,48] and the references therein.

The results of fitting our model to the experimental data are shown in Figs. 4(a), 4(d), and 4(e). In the case of the undoped YIG film, the $M$ vs $H$ curves obtained from the model show a linear slope (positive for $d$ sites, negative for $a$ sites) up to about $200 \mathrm{mT}$, and then they saturate for higher magnetic fields, matching the experimental data perfectly [symbols in Fig. 4(a)]. Configurations of $\mathbf{M}_{\boldsymbol{d}}$ and $\mathbf{M}_{\boldsymbol{a}}$ are schematically represented in the four topmost ellipse-shaped sketches in Fig. 4(a). In this case, the evolution of $\mathbf{M}_{\boldsymbol{d}}$ and $\mathbf{M}_{\boldsymbol{a}}$ with $H$ is precisely what one would expect for antiparallel-coupled $\mathbf{M}_{\boldsymbol{d}} / \mathbf{M}_{\boldsymbol{a}}$ magnetizations with uniaxial anisotropy, provided
TABLE II. Fitting parameters of the magnetization hysteresis loops according to Eq. (5).

\begin{tabular}{lcccc}
\hline \hline Sample & $\begin{array}{c}H_{d}=H_{a} \\
K_{d} / M_{d}(\mathrm{kA} / \mathrm{m})\end{array}$ & $\begin{array}{c}J_{d / a} \\
\left(\mathrm{MJ} / \mathrm{m}^{3}\right)\end{array}$ & $\begin{array}{c}J_{\mathrm{Ce} / d} \\
\left(\mathrm{MJ} / \mathrm{m}^{3}\right)\end{array}$ & $\begin{array}{c}J_{\mathrm{Ce} / a} \\
\left(\mathrm{MJ} / \mathrm{m}^{3}\right)\end{array}$ \\
\hline Ce-YIG(001) & 58 & 4.20 & 0.10 & -0.10 \\
Ce-YIG(111) & 19 & 1.70 & 0.07 & -0.07 \\
\hline \hline
\end{tabular}

that $H$ is not sufficiently large to overcome the exchange coupling field. In these simulations, the exchange interaction between the $a$ and $d$ sublattices denoted by $J_{d / a}$, denoted by $J_{d / a}$ in Eq. (5), is $J_{d / a}=J$ [see Fig. 4(a), solid lines]. When we apply $\mathbf{H}$ perpendicular to the film plane, the magnetization vectors of both sublattices rotate coherently in the magnetization reversal process, as shown by the schematic arrows of the upper sketches in Fig. 4(a). At higher fields, $\mathbf{M}_{\boldsymbol{d}}$ is aligned parallel to $\mathbf{H}$, while $\mathbf{M}_{\boldsymbol{a}}$ points to the opposite direction.

However, as described above, the $M_{d}(H)$ and $M_{a}(H)$ curves of the Fe ions for Ce-doped YIG films have an unusual change in the slope that causes the overshoot of the magnetization revealed in the XMCD loops displayed in Figs. 4(b) and 4(c). Given that the contribution of $M_{\mathrm{Ce}}$ to the total moment is very small, we made the reasonable assumption that the unusual slope is solely attributed to the decrease of the antiparallel $M_{d} / M_{a}$ coupling. Bearing this in mind, we simulated a pair of curves by using $J_{d / a}=\frac{J}{3}$ as the value for the exchange coupling [see Fig. 4(a)], i.e., reducing $J_{d / a}$ to one third of the value used to simulate the experimental data of the undoped YIG film. We attribute the decrease of $J_{d / a}$ to the multivalence state redistribution inferred from the analysis of the Fe-XAS and XMCD spectra, which significantly alters the $\mathrm{Fe}^{3+}(d)-\mathrm{O}-\mathrm{Fe}^{3+}(a)$ interactions. As demonstrated in Fig. 4(a), after reducing the value of $J_{d / a}$, the shape of the respective simulated curves reproduces very well the atypical shape of the Fe XMCD loops of Ce-doped samples. The XMCD loops of Ce-YIG films [see Figs. 4(b) and 4(c)] were also modeled according to Eq. (5); the resulting curves, calculated employing the parameters displayed in Table II, are plotted in Figs. 4(d) and 4(e). Note that the undoped YIG sample could fit to any value $J_{d / a}>0.48 \mathrm{MJ} / \mathrm{m}^{3}$ to match the experimental XMCD loop. Therefore, our assumption is that the value of $J_{d / a}$ for the YIG film is significantly larger than those for (001)- and (111)-oriented Ce-YIG films, which, according to the fittings, have values of $J_{d / a}=4.20 \mathrm{MJ} / \mathrm{m}^{3}$ and $1.70 \mathrm{MJ} / \mathrm{m}^{3}$, respectively. Note also that whereas the fitting $M_{d}(H)$ and $M_{a}(H)$ curves reproduce rather well the experiment, the calculated $M_{\mathrm{Ce}}$ ones show certain negative (positive) slope for high negative (positive) fields absent in the experimental $M_{\mathrm{Ce}}$ curves. We performed simulations (not shown) which attested that this slope could be greatly reduced by considering, e.g., a non-negligible anisotropy (with the in-plane easy magnetization direction) of the $\mathrm{Ce}^{3+}$ ions, together with different $J_{a / \mathrm{Ce}}$ and $J_{d / \mathrm{Ce}}$ values and/or signs. Nevertheless, due to the greatly increased number of free fitting parameters needed to obtain such (only qualitative) improvements, we chose not to present these simulations. 
The effects of the reduced exchange coupling on the sublattice magnetization loops can be understood intuitively as indicated in the two sketches depicted at the bottom of Fig. 4(a). To have a visual understanding of these effects, we also include an animated video that describes schematically the magnetization reversal in the case of reduced, by the incorporation of $\mathrm{Ce}$, exchange coupling [49]. Briefly, for small values of $H$, the $a$ - and $d$-site magnetization components are practically perfectly antiparallel, as observed for the undoped YIG. However, at higher fields, the competition between the Zeeman and exchange coupling energies leads to dissimilar rotations of $\mathbf{M}_{\boldsymbol{d}}$ and $\mathbf{M}_{\boldsymbol{a}}$, as depicted in the bottom sketches of Fig. 4(a). Focusing on the $d$ sublattice, the evolution of $\mathbf{M}_{\boldsymbol{d}}$ for weak $J_{d / a}$ coupling can be described by the energy term $-\mathbf{M}_{\boldsymbol{d}} \cdot \mathbf{H}_{d}^{\text {eff }}$, where the effective field is given by $\mathbf{H}_{d}^{\text {eff }}=\mathbf{H}+k\left|\mathbf{M}_{a}\right|$, with $k=J_{d / a} /\left(M_{d} M_{a}\right)$. As $H$ increases, $\mathbf{M}_{\boldsymbol{a}}$, which is antiparallel to $\mathbf{M}_{\boldsymbol{d}}$, starts to rotate towards $\mathbf{H}$. As a result, the projection of $\mathbf{M}_{\boldsymbol{a}}$ along $\mathbf{H}$ decreases with the increase in $H$, so that the value of $\mathbf{H}_{d}^{\text {eff }}$ may actually decrease, thus inducing a decrease in the projection of $\mathbf{M}_{\boldsymbol{d}}$ along the $H$ direction at high enough fields, as observed in the unusual XMCD loops of the Ce-YIG films [see Fig. 4(a)]. A similar analysis can be done by interchanging the roles of $\mathbf{M}_{\boldsymbol{a}}$ and $\mathbf{M}_{\boldsymbol{d}}$. We therefore conclude that the observed decreasing high-field slopes of the XMCD loops obtained from $\mathrm{Fe} a$ sites and $d$ sites are due to a weakening of the exchange coupling $J_{d / a}$ driven by a charge-transfer process triggered by doping YIG with Ce.

We discuss in the following the microscopic mechanisms that may lead to the reduction of the exchange coupling in the Ce-doped YIG films. In magnetic insulators, it is well known that the exchange interaction is predominantly defined by the so-called superexchange interaction, which is due to the overlap of the electronic orbitals from the magnetic cations via intermediate ligands. Starting from the undoped YIG system, the $\mathrm{FeO}_{6}$ octahedra are corner linked to the neighboring $\mathrm{FeO}_{4}$ tetrahedral sites via the $\mathrm{O}$ ligands [see Fig. 1(b)], with all the Fe $3 d$ orbitals being half occupied in a high-spin configuration, which results in a quenching of the orbital moment (see Table I for the undoped YIG case).

In YIG, the $\mathrm{Fe}(d)-\mathrm{O}-\mathrm{Fe}(a)$ bond angle between these two crystallographically inequivalent $\mathrm{Fe}$ sites is about $\sim 126^{\circ}$ [see angle $\theta$ in Fig. 1(b)] [50], and their magnetic coupling is mostly accomplished by the superexchange interaction via the $2 p$ orbitals of the common $\mathrm{O}$ ligand atom. According to the Goodenough-Kanamori-Anderson (GKA) rules, [51], $3 d^{5}-3 d^{5}$ superexchange interactions via a $180^{\circ}$ bond angle would stipulate an antiferromagnetic (AFM) coupling. Because in YIG the metal-oxygen-metal bond angle connecting tetrahedral and octahedral sites is $\sim 126^{\circ}$ [50], the AFM interaction, although weaker than for $180^{\circ}$, will produce an antiparallel coupling of $\mathrm{Fe}^{3+}(d)-\mathrm{Fe}^{3+}(a)$, which thus gives rise to the observed ferrimagnetic arrangement of tetrahedral and octahedral sublattices.

Now, turning to the case of Ce-doped YIG, our experimental data point to charge transfer promoted from $\mathrm{Ce}$ to $\mathrm{Fe}$, which can cause a double occupancy in one of the two $e_{g}$ levels at the $\mathrm{Fe}^{2+}\left(3 d^{6}\right)$ ions on the tetrahedral $d$ sites. In this scenario, the additional electron coming from the charge transfer will occupy available spin-down states of the low-lying $e_{g}$ orbitals of either $d_{x^{2}-y^{2}}$ or $d_{z^{2}}$ symmetry of $3 d^{6}\left(e_{g}^{1 \downarrow}\right)$

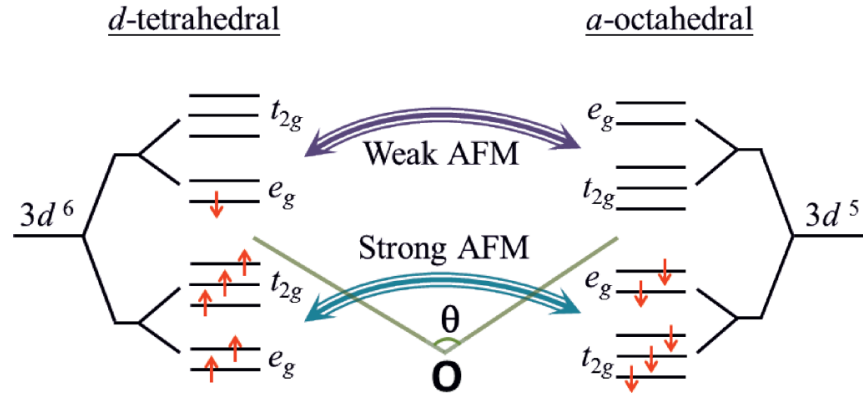

FIG. 5. Schematic diagram of the energy levels at $\mathrm{Fe}\left(3 d^{6}\right)-\mathrm{O}-\mathrm{Fe}\left(3 d^{5}\right)$ superexchange coupling. $\theta$ is the angle between the $d$-Fe and $a$-Fe sites. The $d^{5}-d^{5}$ coupling is a strong antiferromagnetic while the spin-down electron of $d^{6}$ weakens the coupling by freely moving to empty spin-up states of $d^{5}$ orbitals.

(see Fig. 5). Therefore, the strength of the newly established $3 d^{6}\left(t_{2 g}^{3 \uparrow} e_{g}^{2 \uparrow} e_{g}^{1 \downarrow}\right)-\mathrm{O}-3 d^{5}\left(t_{2 g}^{3 \uparrow} e_{g}^{2 \uparrow}\right)$ interaction must be modified. Because, for $\mathrm{Fe}-\mathrm{O}-\mathrm{Fe}$ bonds differing from $180^{\circ}$ or $90^{\circ}$, the contributions of different $3 d$ orbitals to the bond become somehow mixed, in practice the GKA rules cannot be simply used to predict the strength of the new interaction. However most likely the contribution from the transferred $3 d^{6}\left(e_{g}^{1 \downarrow}\right)$ electron will reduce the strength of the $\mathrm{AF}$ coupling and the magnitude of net exchange constant $J_{d / a}$ arising from the superexchange $\mathrm{Fe}^{2+/ 3+}(d) \leftrightarrow \mathrm{Fe}^{3+}(a)$ interaction (see the sketch in Fig. 5). In other words, charge doping at Fe ions arising from $\mathrm{Ce}^{4+}$ substitution explains the reduction of the $J_{d / a}$ exchange coupling constant.

\section{SUMMARY}

To sum up, the spin dynamics of the Ce-YIG thin films from the FMR measurements reveals that our films exhibit a magnetic damping comparable to magnetic metals $(\alpha \sim$ $2-4 \times 10^{-2}$ ). XAS and XMCD experiments were used to probe site-specific sublattice contributions to the magnetism of Ce-doped YIG and to have a direct observation of the valence states of $\mathrm{Ce}$ and $\mathrm{Fe}$ ions. Our experimental results were consistent with the emergence of a charge transfer from $\mathrm{Ce}^{3+}\left(4 f^{1}\right)+\mathrm{Fe}^{3+}\left(3 d^{5}\right) \rightarrow \mathrm{Ce}^{4+}\left(4 f^{0}\right)+\mathrm{Fe}^{2+}\left(3 d^{6}\right)$ into the tetrahedral $d$ sites of Fe driven by the Ce doping into YIG. As a result, a large fraction of $\mathrm{Ce}^{4+}$ ions appears, and on the other hand, the charge transfer also induces a partial reduction of $\mathrm{Fe}^{3+}$ into $\mathrm{Fe}^{2+}\left(3 d^{6}\right)$, which causes a reduction of the overall magnetic moment. Furthermore, the value of the exchange coupling constant $J_{d / a}$ between $\mathrm{Fe}$ ions at $d$ (tetrahedral) and $a$ (octahedral) sites is reduced, which also entails a reduction of the magnetic coupling between both sublattices. This produces visible features in the magnetization process, noticeable at low magnetic fields. The last observation is responsible for the occurrence of atypical XMCD magnetization curves for the $\mathrm{Fe}$ ions, caused by an overshoot of the magnetization and a subsequent reduction of the $\mathrm{Fe}$-sublattice magnetization with increased applied magnetic fields. The atypical signatures observed for the site-specific magnetic contributions in doped YIG may prove relevant for a better understanding of the magneto-optical properties of these compounds. 
At first glance, it may seem startling to observe a detrimental effect of the $\mathrm{Ce}$ incorporation on the magnetic properties, e.g., a reduction in the exchange coupling and magnetization, in a system which is known to have a significantly larger magneto-optical activity than the undoped YIG. We should bear in mind, however, that, although linear magneto-optical effects are proportional to the magnetization, the constant of proportionality depends decisively on the details of the electronic band structure and the allowed optical transitions. More precisely, the charge transfer from Ce to Fe plays a crucial role in understanding the enhanced magneto-optical response in the $\mathrm{Ce}-\mathrm{YIG}$ as the response can be explained by the emergence of new optical transitions [especially $\mathrm{Ce}^{3+} \rightarrow$ $\mathrm{Fe}^{2+}(d)$ transitions], which are absent in the parent undoped YIG, involving ions ( $\mathrm{Ce}$ in this case) with large spin-orbit coupling. Beyond the optical applications, our findings can also be important for other applications in which, e.g., spin waves are exploited. In this case, site-specific doping may reveal itself to be an important tool kit for engineering the dynamic magnetic properties based on the demands of potential applications.

\section{ACKNOWLEDGMENTS}

This work was supported by the Spanish government by the MAT2014-56063-C2-1-R and Severo Ochoa SEV-20150496 Projects, the Generalitat de Catalunya (2014 SGR 734 Project), and the Brazilian agency CAPES (BEX 0298/201508 Project). B.C. acknowledges Grant No. FPI BES-2012059023, and R.C. thanks CNPq, Brazil, for his fellowship. We also acknowledge Dr. F. Sánchez for scientific guidance and discussions on materials issues. XAS and XMCD experiments were performed at the Boreas beamline of the Synchrotron Light Facility ALBA with the collaboration of ALBA staff. We acknowledge M. Opel and R. Gross for providing us with the undoped YIG sample.
[1] K. Uchida, J. Xiao, H. Adachi, J. Ohe, S. Takahashi, J. Ieda, T. Ota, Y. Kajiwara, H. Umezawa, H. Kawai, G. E. W. Bauer, S. Maekawa, and E. Saitoh, Nat. Mater. 9, 894 (2010).

[2] C. W. Sandweg, Y. Kajiwara, K. Ando, E. Saitoh, and B. Hillebrands, Appl. Phys. Lett. 97, 252504 (2010).

[3] L. H. Vilela-Leão, C. Salvador, A. Azevedo, and S. M. Rezende, Appl. Phys. Lett. 99, 102505 (2011).

[4] G. F. Dionne, Magnetic Oxides (Springer, New York, 2009), pp. 37-106.

[5] F. D. M. Haldane and S. Raghu, Phys. Rev. Lett. 100, 013904 (2008).

[6] A. V. Chumak, V. S. Tiberkevich, A. D. Karenowska, A. A. Serga, J. F. Gregg, A. N. Slavin, and B. Hillebrands, Nat. Commun. 1, 141 (2010).

[7] M. Gomi, H. Furuyama, and M. Abe, J. Appl. Phys. 70, 7065 (1991).

[8] M. C. Sekhar, M. R. Singh, S. Basu, and S. Pinnepalli, Opt. Express 20, 9624 (2012).

[9] M. C. Onbasli, L. Beran, M. Zahradník, M. Kučera, R. Antoš, J. Mistrík, G. F. Dionne, M. Veis, and C. A. Ross, Sci. Rep. 6, 23640 (2016).

[10] L. Bi, J. Hu, P. Jiang, D. H. Kim, G. F. Dionne, L. C. Kimerling, and C. A. Ross, Nat. Photonics 5, 758 (2011).

[11] T. Goto, M. C. Onbaşl1, and C. A. Ross, Opt. Express 20, 28507 (2012).

[12] S. Ghosh, S. Keyvavinia, W. Van Roy, T. Mizumoto, G. Roelkens, and R. Baets, Opt. Express 20, 1839 (2012).

[13] T. Mizumoto, R. Takei, and Y. Shoji, IEEE J. Quantum Electron. 48, 252 (2012).

[14] X. Liang, J. Xie, L. Deng, and L. Bi, Appl. Phys. Lett. 106, 052401 (2015).

[15] M. Kucera, J. Bok, and K. Nitsch, Solid State Commun. 69, 1117 (1989).

[16] Y. Xu, J. H. Yang, and X. J. Zhang, Phys. Rev. B 50, 13428 (1994).

[17] B. Casals, M. Espínola, R. Cichelero, S. Geprägs, M. Opel, R. Gross, G. Herranz, and J. Fontcuberta, Appl. Phys. Lett. 108, 102407 (2016).
[18] R. L. Streever and P. J. Caplan, Phys. Rev. B 4, 2881 (1971).

[19] C. Hauser, T. Richter, N. Homonnay, C. Eisenschmidt, M. Qaid, H. Deniz, D. Hesse, M. Sawicki, S. G. Ebbinghaus, and G. Schmidt, Sci. Rep. 6, 20827 (2016).

[20] A. Sposito, T. C. May-Smith, G. B. G. Stenning, P. A. J. de Groot, and R. W. Eason, Opt. Mater. Express 3, 624 (2013).

[21] A. Barla, J. Nicolas, D. Cocco, S. M. Valvidares, J. HerreroMartin, P. Gargiani, J. Moldes, C. Ruget, E. Pellegrin, and S. Ferrer, J. Synchrotron Radiat. 23, 1507 (2016).

[22] E. Stavitski and F. M. F. de Groot, Micron 41, 687 (2010).

[23] See Supplemental Material at http://link.aps.org/supplemental/ 10.1103/PhysRevB.96.014433 for a discussion of x-ray diffraction analysis of Ce-YIG thin films and the $\mathrm{x}$-ray spectroscopy of the $\mathrm{Y} L_{2,3}$ absorption edges.

[24] T.-C. Mao and J.-C. Chen, J. Magn. Magn. Mater. 302, 74 (2006).

[25] S. M. Shahrokhvand, A. S. H. Rozatian, M. Mozaffari, S. M. Hamidi, and M. M. Tehranchi, J. Phys. D 45, 235001 (2012).

[26] S. S. Kalarickal, P. Krivosik, M. Wu, C. E. Patton, M. L. Schneider, P. Kabos, T. J. Silva, and J. P. Nibarger, J. Appl. Phys. 99, 093909 (2006).

[27] A. Kehlberger, K. Richter, M. C. Onbasli, G. Jakob, D. H. Kim, T. Goto, C. A. Ross, G. Götz, G. Reiss, T. Kuschel, and M. Kläui, Phys. Rev. Appl. 4, 014008 (2015).

[28] F. Michael, Rep. Prog. Phys. 61, 755 (1998).

[29] J. Walowski, M. D. Kaufmann, B. Lenk, C. Hamann, J. McCord, and M. Münzenberg, J. Phys. D 41, 164016 (2008).

[30] C. L. Melcher, S. Friedrich, S. P. Cramer, M. A. Spurrier, P. Szupryczynski, and R. Nutt, IEEE Trans. Nucl. Sci. 52, 1809 (2005).

[31] A. Rogalev, J. Goulon, F. Wilhelm, C. Brouder, A. Yaresko, J. Ben Youssef, and M. V. Indenbom, J. Magn. Magn. Mater. 321, 3945 (2009).

[32] E. Pellegrin, M. Hagelstein, S. Doyle, H. O. Moser, J. Fuchs, D. Vollath, S. Schuppler, M. A. James, S. S. Saxena, L. Niesen, O. Rogojanu, G. A. Sawatzky, C. Ferrero, M. Borowski, O. Tjernberg, and N. B. Brookes, Phys. Status Solidi B 215, 797 (1999). 
[33] V. Hari Babu, R. K. Govind, K.-M. Schindler, M. Welke, and R. Denecke, J. Appl. Phys. 114, 113901 (2013).

[34] S. W. Yu, M. H. Carpenter, F. Ponce, S. Friedrich, J. S. Lee, P. Olalde-Velasco, W. L. Yang, and D. Åberg, J. Phys. Condens. Matter 27, 239601 (2015).

[35] X. Guo, A. H. Tavakoli, S. Sutton, R. K. Kukkadapu, L. Qi, A. Lanzirotti, M. Newville, M. Asta, and A. Navrotsky, Chem. Mater. 26, 1133 (2014).

[36] For the LFM calculations, final Slater integrals $\mathrm{F}(p d)$ and $\mathrm{G}(p d)$ of $77 \%$ and $74 \%$, respectively, were chosen for both the $\mathrm{Fe}$ ( $a$ octahedral and $d$ tetrahedral) sites after reducing them to a standard reduction of $80 \%$ to the Hartree-Fock values. We have varied the Slater integral of $\mathrm{F}(d d)$ for $a \mathrm{Fe}^{3+}(68 \%), d$ $\mathrm{Fe}^{3+}(60 \%)$, and $d \mathrm{Fe}^{2+}(76 \%)$ sites separately. In the $C_{4}$ crystal symmetry, $10 \mathrm{Dq}$ values of 1.6 and $-0.8 \mathrm{eV}$ were used for the $a$ and $d$ sites, respectively. The $M$ value of the $a \mathrm{Fe}^{3+}$ site $(-12 \mathrm{meV})$ was chosen to be opposite to the $d \mathrm{Fe}^{3+}$ sites $(18 \mathrm{meV})$ or $d \mathrm{Fe}^{2+}$ sites $(9 \mathrm{meV})$ as the magnetic moments of Fe ions at $a$ sites and $d$ sites are antiparallel to each other. All the spectra were further broadened by a Lorentzian half width of 0.16 and $0.4 \mathrm{eV}$ after the energy split of $709 \mathrm{eV}$. The instrumental broadening was also accounted for by taking a Gaussian broadening of $0.12 \mathrm{eV}$.

[37] B. T. Thole, P. Carra, F. Sette, and G. van der Laan, Phys. Rev. Lett. 68, 1943 (1992).

[38] P. Carra, B. T. Thole, M. Altarelli, and X. Wang, Phys. Rev. Lett. 70, 694 (1993).

[39] C. T. Chen, Y. U. Idzerda, H.-J. Lin, N. V. Smith, G. Meigs, E. Chaban, G. H. Ho, E. Pellegrin, and F. Sette, Phys. Rev. Lett. 75, 152 (1995).
[40] D. J. Huang, C. F. Chang, H. T. Jeng, G. Y. Guo, H. J. Lin, W. B. Wu, H. C. Ku, A. Fujimori, Y. Takahashi, and C. T. Chen, Phys. Rev. Lett. 93, 077204 (2004).

[41] C. Piamonteze, P. Miedema, and F. M. F. de Groot, Phys. Rev. B 80, 184410 (2009).

[42] T. Okane, Y. Takeda, H. Yamagami, A. Fujimori, Y. Matsumoto, N. Kimura, T. Komatsubara, and H. Aoki, Phys. Rev. B 86, 125138 (2012).

[43] G. van der Laan and B. T. Thole, Phys. Rev. B 53, 14458 (1996).

[44] P. Novák, J. Englich, H. Štěpánková, J. Kohout, H. Lütgemeier, K. Wagner, and W. Tolksdorf, Phys. Rev. Lett. 75, 545 (1995).

[45] E. Popova, N. Keller, F. Gendron, M. Guyot, M.-C. Brianso, Y. Dumond, and M. Tessier, J. Appl. Phys. 90, 1422 (2001).

[46] C. L. Jermain, S. V. Aradhya, N. D. Reynolds, R. A. Buhrman, J. T. Brangham, M. R. Page, P. C. Hammel, F. Y. Yang, and D. C. Ralph, Phys. Rev. B 95, 174411 (2017)

[47] J. Geshev, A. D. C. Viegas, and J. E. Schmidt, J. Appl. Phys. 84, 1488 (1998).

[48] J. Geshev, L. G. Pereira, J. E. Schmidt, and M. Mikhov, J. Appl. Phys. 90, 6243 (2001).

[49] See the video for the magnetization reversal process of the $\operatorname{Fe}(a)$ and $\operatorname{Fe}(d)$ sites in the Supplemental Material [23].

[50] M. A. Gilleo, in Handbook of Ferromagnetic Materials (Elsevier, Amsterdam, 1980), Vol. 2, pp. 1-53.

[51] J. B. Goodenough, Magnetism and the Chemical Bond (Wiley Interscience, New York, 1963). 